\title{
A LOGARITHMIC EFFICIENT ESTIMATOR \\ OF THE PROBABILITY OF RUIN WITH RECUPERATION \\ FOR SPECTRALLY NEGATIVE LEVY RISK PROCESSES
}

\author{
Riccardo Gatto
}

Submitted: April 2014

Revised: July 2014

\begin{abstract}
This article provides an importance sampling algorithm for computing the probability of ruin with recuperation of a spectrally negative Lévy risk process with light-tailed downwards jumps. Ruin with recuperation corresponds to the following double passage event: for some $t \in(0, \infty)$, the risk process starting at level $x \in[0, \infty)$ falls below the null level during the period $[0, t]$ and returns above the null level at the end of the period $t$. The proposed Monte Carlo estimator is logarithmic efficient, as $t, x \rightarrow \infty$, when $y=t / x$ is constant and below a certain bound.
\end{abstract}

\section{Key words and phrases}

Esscher approximation; exponential tilt; Monte Carlo simulation; importance sampling; Legendre-Fenchel transform.

The author is grateful to an anonymous Referee for several important suggestions and corrections. 2010 Mathematics Subject Classification: 65C05, 60G51.

Address: Institute of Mathematical Statistics and Actuarial Science, Department of Mathematics and Statistics, University of Bern, Alpeneggstrasse 22, 3012 Bern, Switzerland.

Email: gatto@stat.unibe.ch. 


\section{Introduction}

Stochastic simulation is a practical technique for computing probabilities of rare events related to stochastic processes, like the payoff probability of a financial option, the probability that a queue exceeds a certain level or the probability of ruin of the insurer's risk process. In these situations, it is convenient to shift the sampling distribution in order to thwart the rarity of the event to simulate. This is called importance sampling which, in the present context, originates from Siegmund (1976). Two main contributions in the context of probabilities of insurer's ruin are Asmussen (1985) and Section X.4 of Asmussen (2000). Two general references are Asmussen and Glynn (2007) and Bucklew (2004). Gatto (2014) provides importance sampling algorithms for finite and infinite time probabilities of ruin as well as for the probability of the ruin past a finite time horizon, in the context of spectrally negative Lévy processes. This article provides an importance sampling algorithm for the probability of ruin with recuperation for spectrally negative Lévy processes with light-tailed downwards jumps. It is the probability that the risk process starting at level $x \geq 0$ falls below the null level during the time horizon $[0, t]$, for some $t \in(0, \infty)$, and ends at or above the null level at time $t$. The suggested Monte Carlo algorithm is logarithmic efficient, as $t, x \rightarrow \infty$, when $y \stackrel{\text { def }}{=} t / x$ is fixed and bounded from above.

In this article, the fluctuation of the capital of the insurance is represented by the general spectrally negative Lévy risk process $Y$ in $\mathbb{R}^{[0, \infty)}$ defined by

$$
Y_{t}=x-S_{t}, \forall t \geq 0
$$

where $x \geq 0$ is the initial capital and $S=\left\{S_{t}\right\}_{t \geq 0}$ is the compensated loss Lévy process, which represents the aggregate claim amount minus the aggregate income. The process $S$ allows only for positive jumps, which represent individual claim amounts. The literature on Lévy risk processes has become important. We can for example mention Klüppelberg et al. (2004), Avram et al. (2007), Kyprianou and Palmowski (2007), Biffis and Morales (2010), etc. The business risk inherent to (1) can be represented by various types probabilities of ruin. Let us first define the time of ruin as

$$
T_{x}= \begin{cases}\inf \left\{t \in(0, \infty) \mid Y_{t}<0\right\}, & \text { if the infimum exists } \\ \infty, & \text { otherwise }\end{cases}
$$

The probability of ruin within the finite time horizon $[0, t]$ is defined by

$$
\psi(x, t)=\mathrm{P}\left[T_{x} \leq t\right]
$$

where here and in the following $t \in(0, \infty)$ is fixed. So $\psi(x, t)$ is the probability that $Y$ falls below the null level prior to time $t$. The probability of ruin within the infinite time 
horizon is defined by $\psi(x)=\mathrm{P}\left[T_{x}<\infty\right]=\lim _{t \rightarrow \infty} \psi(x, t)$. It is the probability that $\left\{Y_{t}\right\}_{t \geq 0}$ ever falls below zero. We focus on the probability of ruin with recuperation, which is the probability of ruin within the finite time horizon $[0, t]$ and recuperation at time $t$, which means $Y_{t} \geq 0$. Thus, this probability is given by

$$
\check{\psi}(x, t)=\mathrm{P}\left[T_{x} \leq t \wedge Y_{t} \geq 0\right]
$$

Clearly,

$$
\begin{aligned}
\psi(x, t) & =\mathrm{P}\left[T_{x} \leq t \wedge Y_{t} \geq 0\right]+\mathrm{P}\left[T_{x} \leq t \wedge Y_{t}<0\right] \\
& =\mathrm{P}\left[T_{x} \leq t \wedge Y_{t} \geq 0\right]+\mathrm{P}\left[Y_{t}<0\right] \\
& =\check{\psi}(x, t)+\zeta(x, t)
\end{aligned}
$$

where $\zeta(x, t)=\mathrm{P}\left[S_{t}>x\right]$. Thus the quantity we suggest computing by importance sampling is re-expressed as

$$
\check{\psi}(x, t)=\psi(x, t)-\zeta(x, t) .
$$

The remaining part of this article has the following structure. Section 2 reviews the basic theory of Lévy processes and gives the assumptions considered in our model. Section 3 presents the proposed importance sampling estimator to (2) together with a proof of logarithmic efficiency. At the end, Section 4 contains two remarks relating the suggested estimator with two alternative existing methods.

\section{Lévy processes and change of measure}

This section summarizes the important facts for this article of the theory Lévy processes. Two general references are Applebaum (2004) and Bertoin (1996). Because important claim amounts lead to upward jumps in the insurer's loss process $S$, it is assumed that $S$ is a spectrally positive Lévy process, as defined below. The Laplace exponent of any Lévy process $L$ on $\mathbb{R}^{[0, \infty)}$ is defined as

$$
\kappa(v)=\log \mathrm{E}\left[\mathrm{e}^{v L_{1}}\right],
$$

$\forall v \in \mathbb{R}$ s.t. $\kappa(v)<\infty$. It turns out that $t \kappa$ is the cumulant generating function of $L_{t}$, $\forall t \geq 0$. The Lévy-Khintchine representation is given by

$$
\kappa(v)=\gamma v+\frac{1}{2} \sigma^{2} v^{2}+\int_{\mathbb{R}}\left(\mathrm{e}^{v x}-1-v x \mathrm{I}\{|x|<1\}\right) \mathrm{d} \nu(x),
$$


where $\gamma \in \mathbb{R}, \sigma>0$ and $\nu$ is a Lévy measure, i.e. a measure on $(\mathbb{R} \backslash\{0\}, \mathcal{B}(\mathbb{R} \backslash\{0\}))$ which satisfies

$$
\int_{\mathbb{R}}\left(1 \wedge x^{2}\right) \mathrm{d} \nu(x)<\infty
$$

The characteristic triplet of $(5)$ or of $L$ is $\left(\gamma, \sigma^{2}, \nu\right)$. An a.s. nondecreasing Lévy process is called subordinator. The Lévy process $L$ can be decomposed as the sum of: a constant drift (i.e. a linear function), a Wiener process and a jump process $J$ in $\mathbb{R}^{[0, \infty)}$. The jump process $J$ is the sum of a process displaying infinitely many jumps of vanishing magnitude per unit of time, plus a second process displaying finitely many jumps of substantial magnitude per unit of time. The process $J$ is characterized by the Lévy measure $\nu$, which represents the intensity of the jumps. The Lévy process $L$ is called spectrally positive if it is not a subordinator and $\nu\left[\mathbb{R}_{-}\right]=0$. Further, $L$ is called spectrally negative if $-L$ is spectrally positive. The jumps of a spectrally positive (negative) Lévy process can only be directed upwards (downwards).

We now consider $S$ with Laplace exponent (5). Let $v \in \mathbb{R}$, then $\kappa(v)<\infty$ if

$$
\int_{(-\infty,-1]}\left|\mathrm{e}^{v x}-1\right| \mathrm{d} \nu(x)+\int_{(-1,1)}\left|\mathrm{e}^{v x}-1-v x\right| \mathrm{d} \nu(x)+\int_{[1, \infty)}\left|\mathrm{e}^{v x}-1\right| \mathrm{d} \nu(x)<\infty .
$$

Consider

$$
\chi_{1}(v) \stackrel{\text { def }}{=} \int_{(-\infty,-1]} \mathrm{e}^{v x} \mathrm{~d} \nu(x) \text { and } \chi_{2}(v) \stackrel{\text { def }}{=} \int_{[1, \infty)} \mathrm{e}^{v x} \mathrm{~d} \nu(x)
$$

The following simplifications are consequences of (6). As $\left|\mathrm{e}^{v x}-1-v x\right| \leq \mathrm{e} v^{2} x^{2} / 2, \forall x \in$ $(-1,1)$ (from Taylor expansion), the second integral in $(7)$ is always finite. If $v>0$, then $\chi_{2}(v)<\infty$ is equivalent to the finiteness of the third integral in (7). If $v<0$, then $\chi_{1}(v)<\infty$ is equivalent to the finiteness of the first integral in (7). Therefore, $\kappa(v)<\infty$ is equivalent to $\chi_{2}(v)<\infty$, if $v>0$, and to $\chi_{1}(v)<\infty$, if $v<0$. Because $S$ is spectrally positive, $\chi_{1}(v)=0, \forall v \in \mathbb{R}$, and therefore we obtain: if $v<0$, then $\kappa(v)<\infty$, and if $v>0$, then $\kappa(v)<\infty \Leftrightarrow \chi_{2}(v)<\infty$. The fact $\chi_{2}(v)<\infty$, for some $v>0$, is referred as light-tailness of upwards jumps of the spectrally positive process.

We assume that $\exists s \in(0, \infty]$ s.t. $\lim _{v \rightarrow s, v<s} \kappa(v)=\infty$ and $\kappa(s-\varepsilon)<\infty, \forall \varepsilon>0$, which is referred as the steepness of the Laplace exponent. This steepness can be simplified to

$$
\exists s \in(0, \infty] \text { s.t. } \quad \lim _{v \rightarrow s, v<s} \chi_{2}(v)=\infty \text { and } \chi_{2}(s-\varepsilon)<\infty, \forall \varepsilon>0,
$$

which is in fact stronger than light-tailness of upwards jumps. We further assume

$$
\mu \stackrel{\text { def }}{=} \mathrm{E}\left[S_{1}\right]=\gamma+\int_{(-\infty,-1] \cup[1, \infty)} x \mathrm{~d} \nu(x)<0
$$


which is referred as net profit condition.

We now consider the spectrally positive Lévy loss process $S$ over the filtered probability space $\left(\Omega, \mathcal{F},\left\{\mathcal{F}_{t}\right\}_{t \geq 0}, \mathrm{P}\right)$. The time of ruin $T_{x}$ is a stopping time of $\left\{\mathcal{F}_{t}\right\}_{t \geq 0}$ and we define $\mathcal{F}_{T_{x}}=\left\{A \in \mathcal{F} \mid A \cap\left\{T_{x} \leq t\right\} \in \mathcal{F}_{t}, \forall t \geq 0\right\}$. Let $\theta \in \mathbb{R}$ s.t. $\kappa(\theta)<\infty$. Assume there exists an equivalent probability measure $\mathrm{P}_{\theta}$ over $\left(\Omega, \mathcal{F},\left\{\mathcal{F}_{t}\right\}_{t \geq 0}\right)$ which transforms the Laplace exponent (4) to $\kappa_{\theta}(v) \stackrel{\text { def }}{=} \log \mathrm{E}_{\theta}\left[\mathrm{e}^{v S_{1}}\right]=\kappa(\theta+v)-\kappa(\theta), \forall v \in \mathbb{R}$ s.t. $\kappa(\theta+v)<\infty$, where $\mathrm{E}_{\theta}$ denotes the expectation under $\mathrm{P}_{\theta}$. Steepness of the Laplace exponent implies $\exists \theta, v>0$ s.t. $\kappa_{\theta}(v)<\infty$. The measure $\mathbf{P}_{\theta}$ is the exponential tilt of $\mathbf{P}$ and it easily seen that the class of Lévy processes is algebraically closed under exponential tilting. Precisely, under $\mathrm{P}_{\theta}, S$ remains a Lévy process and it has characteristic triplet $\left(\gamma_{\theta}, \sigma_{\theta}^{2}, \nu_{\theta}\right)$ given by

$$
\gamma_{\theta}=\gamma+\sigma^{2} \theta+\int_{(-1,1)} x\left(\mathrm{e}^{\theta x}-1\right) \mathrm{d} \nu(x), \sigma_{\theta}^{2}=\sigma^{2} \text { and } \mathrm{d} \nu_{\theta}(x)=\mathrm{e}^{\theta x} \mathrm{~d} \nu(x) .
$$

Thus, either from (9) and (10), or from computing $\kappa_{\theta}^{\prime}(0)=\kappa^{\prime}(\theta)$, we obtain

$$
\mu_{\theta} \stackrel{\text { def }}{=} \mathrm{E}_{\theta}\left[S_{1}\right]=\gamma+\sigma^{2} \theta+\int_{\mathbb{R}} x\left(\mathrm{e}^{\theta x}-\mathrm{I}\{|x|<1\}\right) \mathrm{d} \nu(x) .
$$

If we restrict $\mathrm{P}$ and $\mathrm{P}_{\theta}$ to $\mathcal{F}_{t}$, then the Radon-Nikodym derivative of these restricted measures is $\mathrm{dP} / \mathrm{dP}_{\theta}=\exp \left\{-\theta S_{t}+t \kappa(\theta)\right\}$. This means that, $\forall A \in \mathcal{F}_{t}$,

$$
\mathrm{P}[A]=\mathrm{E}_{\theta}\left[\exp \left\{-\theta S_{t}+t \kappa(\theta)\right\} ; A\right]
$$

Further, if $A \in \mathcal{F}_{T_{x}}$ and $A \subset\left\{T_{x}<\infty\right\}$, then

$$
\mathrm{P}[A]=\mathrm{E}_{\theta}\left[\exp \left\{-\theta S_{T_{x}}+T_{x} \kappa(\theta)\right\} ; A\right]
$$

The adjustment coefficient or Lundberg's exponent $r$ is the positive solution in $v$ of

$$
\kappa(v)=0,
$$

when it exists, and the exponential tilt with $\theta=r$ is called Lundberg conjugation. If the steepness condition (8) holds, then $r$ does exist. In particular, $\mu_{r}=\kappa_{r}^{\prime}(0)=\kappa^{\prime}(r)>0$ implies that $S$ has a positive drift under $\mathrm{P}_{r}$, whence $\mathrm{P}_{r}\left[T_{x}<\infty\right]=1$.

\section{The importance sampling estimator and its loga- rithmic efficiency}

Let $t \geq 0$. We are interested on the event $A=\left\{T_{x} \leq t \wedge Y_{t} \geq 0\right\}$. Clearly, $A=A_{1} \cap A_{2}$, where $A_{1}=\left\{T_{x} \leq t\right\} \in \mathcal{F}_{t}, A_{2}=\left\{Y_{t} \geq 0\right\} \in \mathcal{F}_{t}$ and thus $A \in \mathcal{F}_{t}$. Let $\tilde{\Psi}(x, t, \theta)=$ $\mathrm{I}\left\{T_{x} \leq t \wedge Y_{t} \geq 0\right\} \mathrm{e}^{-\theta S_{t}+t \kappa(\theta)}$, then from (12) follows that $\check{\psi}(x, t, \theta)=\mathrm{E}_{\theta}[\tilde{\Psi}(x, t, \theta)]$ and 
thus $\tilde{\Psi}(x, t, \theta)$ is a Monte Carlo estimator of $\breve{\psi}(x, t, \theta)$. We will however consider another estimator of $\breve{\psi}(x, t, \theta)$, which exploits the decomposition (3). The reason is that no accurate estimate to $\mathrm{E}_{\theta}\left[\tilde{\Psi}^{2}(x, t, \theta)\right]$, in the form of an upper bound or of an asymptotic approximation, which would be required for efficiency assessments using (17), (18) or (19), seems simple to derive. Under these circumstances, let us define

$$
\begin{gathered}
\Psi(x, t, \theta)=\mathrm{I}\left\{T_{x} \leq t\right\} \mathrm{e}^{-\theta S_{T_{x}}+T_{x} \kappa(\theta)}, \\
\mathrm{Z}(x, t, \theta)=\mathrm{I}\left\{Y_{t}<0\right\} \mathrm{e}^{-\theta S_{t}+t \kappa(\theta)}
\end{gathered}
$$

and

$$
\check{\Psi}(x, t, \theta)=\Psi(x, t, \theta)-\mathrm{Z}(x, t, \theta) .
$$

Then from (12) and (13) we have $\check{\psi}(x, t, \theta)=\mathrm{E}_{\theta}[\check{\Psi}(x, t, \theta)]$ and thus $\check{\Psi}(x, t, \theta)$ is a Monte Carlo estimator of $\breve{\psi}(x, t, \theta)$. The corresponding Monte Carlo approximation is given by

$$
\frac{1}{n} \sum_{k=1}^{n} \check{\Psi}_{k}(x, t, \theta) \stackrel{\mathrm{P}_{\theta}}{\longrightarrow} \check{\psi}(x, t, \theta),
$$

where $\check{\Psi}_{1}(x, t, \theta), \ldots, \check{\Psi}_{n}(x, t, \theta)$ are independent generations of $\check{\Psi}(x, t, \theta)$ under $\mathrm{P}_{\theta}$.

The exponential tilting parameter $\theta$ yielding a logarithmic efficient importance sampling estimator of $\breve{\psi}(x, t)$ is provided by Theorem 3.1 below. Just before stating this theorem, we remind that a sequence of rare events $\{A(x)\}_{x \geq 0}$ is characterized by $\theta(x) \stackrel{\text { def }}{=} \mathrm{P}[A(x)] \stackrel{x \rightarrow \infty}{\longrightarrow} 0$. The Monte Carlo estimator $\Theta(x)=\mathrm{I}_{A(x)}$ of $\theta(x), \forall x \geq 0$, is called logarithmic efficient if

$$
\liminf _{x \rightarrow \infty} \frac{|\log \operatorname{var}(\Theta(x))|}{\left|\log \theta^{2}(x)\right|} \geq 1
$$

Note that a stronger and more intuitive efficiency criterion is bounded relative error, that is

$$
\limsup _{x \rightarrow \infty} \frac{\operatorname{var}(\Theta(x))}{\theta^{2}(x)}<\infty
$$

As (17) can be re-expressed as

$$
\forall \varepsilon>0, \limsup _{x \rightarrow \infty} \frac{\operatorname{var}(\Theta(x))}{\theta^{2-\varepsilon}(x)}<\infty
$$

logarithmic efficiency is clearly weaker that bounded relative error. The concept of logarithmic efficiency derives from the large deviations principle; refer to Chapter 5 of Bucklew (2004). 
Theorem 3.1. Assume that the net profit condition (9) and the steepness condition (8) hold. Factorize the finite time horizon as $t=x y$, for $y>0$ fixed, where $x>0$ is the initial capital. Let $v_{y}$ be the solution in $v$ of $\kappa^{\prime}(v)=1 / y$, i.e. of

$$
y\left(\gamma+\sigma^{2} v+\int_{\mathbb{R}} x\left(\mathrm{e}^{v x}-\mathrm{I}\{|x| \leq 1\}\right) \mathrm{d} \nu(x)\right)=1 .
$$

Let

$$
y_{r}=\frac{1}{\mu_{r}}
$$

where $r$ is the adjustment coefficient given by (14) and $\mu_{r}$ is defined by (11). Then

$$
\check{\Psi}\left(x, t, v_{y}\right)=\mathrm{e}^{-v_{y} S_{T_{x}}+T_{x} \kappa\left(v_{y}\right)} \begin{cases}1-\mathrm{e}^{-v_{y}\left(S_{t}-S_{T_{x}}\right)+\left(t-T_{x}\right) \kappa\left(v_{y}\right)}, & \text { if } T_{x} \leq t \text { and } S_{t}>x, \\ 1, & \text { if } T_{x} \leq t \text { and } S_{t} \leq x, \\ 0, & \text { otherwise }\end{cases}
$$

is a logarithmic efficient estimator of $\breve{\psi}(x, t)$, under $\mathrm{P}_{v_{y}}$, as $t, x \rightarrow \infty$, with $y=t / x$ constant and smaller than $y_{r}$, referred as short time horizon.

The root $v_{y}$ has a central role in the saddlepoint approximation of asymptotic analysis and therefore we will call it the saddlepoint of $t \kappa$ at $x$.

The following Lemmas 3.1-3.5 are necessary for the proof of Theorem 3.1.

Lemma 3.2. Assume that the net profit condition (9) and the steepness condition (8) hold. Define

$$
\begin{gathered}
v_{0}=\operatorname{arginf}_{v \in \mathbb{R}} \kappa(v) \text { and } \\
l_{y}=v_{y}-\kappa\left(v_{y}\right) y .
\end{gathered}
$$

Then, in the short time horizon $y<y_{r}$,

$$
v_{0}<r<l_{y}<v_{y}
$$

where $r$ is the adjustment coefficient given by (14), $v_{y}$ is the saddlepoint given by (20) and $y_{r}$ is given by (21).

Note that the Legendre-Fenchel transform (or large deviations rate) of the cumulant generating function $t \kappa$ at $x$ is given by its convex conjugate, i.e. by $\Lambda_{y}(x)=\sup _{v \in(-\infty, s)} v x-t \kappa(v)$, where $s$ is the steepness point of the Laplace exponent given in (8). From steepness, we can simplify it as follows,

$$
\Lambda_{y}(x)=v_{y} x-x y \kappa\left(v_{y}\right)=x\left\{v_{y}-y \kappa\left(v_{y}\right)\right\}=x l_{y} .
$$


Lemma 3.2 is a direct consequence of the convexity of the Laplace exponent $\kappa$. In the following we define the deficit or overshoot at ruin as $D_{x}=-Y_{T_{x}}=S_{T_{x}}-x \geq 0$, on $\left\{T_{x}<\infty\right\}$.

Lemma 3.3. Assume that the net profit condition (9) and the steepness condition (8) hold. Let $\theta>v_{0}$ s.t. $\kappa(\theta)<\infty$, where $v_{0}$ is defined by (22), and let $\tau(\theta)=\sqrt{\kappa^{\prime \prime}(\theta) / \mu_{\theta}^{3}}$. Then,

$$
\frac{S_{t}-t \mu_{\theta}}{\sqrt{t}} \stackrel{\mathrm{d}}{\longrightarrow} \mathcal{N}\left(0, \kappa^{\prime \prime}(\theta)\right) \text {, as } t \rightarrow \infty, \text { under } \mathrm{P}_{\theta},
$$

and

$$
\frac{T_{x}-\frac{x}{\mu_{\theta}}}{\sqrt{x}} \stackrel{\mathrm{d}}{\longrightarrow} \mathcal{N}\left(0, \tau^{2}(\theta)\right) \text {, as } x \rightarrow \infty, \text { under } \mathrm{P}_{\theta},
$$

where $\mu_{\theta}$ is defined by (11).

Proof of Lemma 3.3. The Strong law of large numbers yields

$$
\lim _{t \rightarrow \infty} \frac{S_{t}}{t}=(\frac{S_{\lfloor t\rfloor}}{\lfloor t\rfloor}+\underbrace{\frac{S_{\lfloor t\rfloor}-S_{t}}{\lfloor t\rfloor}}_{\substack{t \rightarrow \infty \\ \longrightarrow}}) \underbrace{\frac{\lfloor t\rfloor}{t}}_{\substack{t \rightarrow \infty \\ \longrightarrow}}=\mu_{\theta}, \mathrm{P}_{\theta^{-}} \text {a.s. }
$$

From the Central limit theorem,

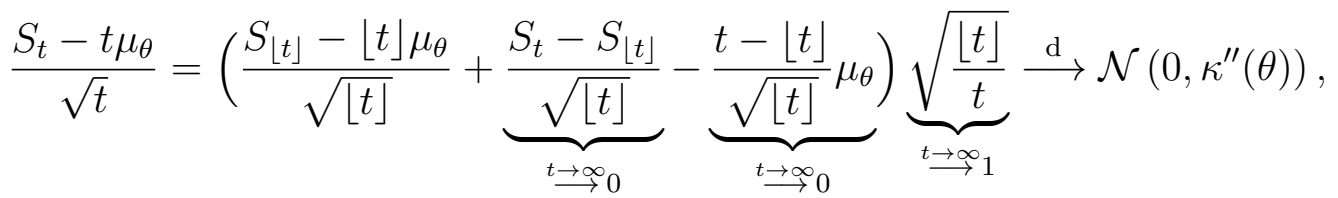

as $t \rightarrow \infty$, under $\mathrm{P}_{\theta}$. Thus (24) holds. From $\mu_{\theta}>0$ follows

$$
\mathrm{P}_{\theta}\left[T_{x}<\infty\right]=1
$$

Also,

$$
\mathrm{P}_{\theta}\left[\lim _{x \rightarrow \infty} T_{x}=\infty\right]=1
$$

is due to the fact that $T_{x}$ is nondecreasing in $x$ and $\mathrm{P}_{\theta^{-}}$a.s. unbounded. From (26), (27), (28) and from $D_{x}=\mathrm{o}(x)$, as $x \rightarrow \infty, \mathrm{P}_{\theta^{-}}$-a.s., we find

$$
\frac{1}{\mu_{\theta}}=\lim _{t \rightarrow \infty} \frac{t}{S_{t}}=\lim _{x \rightarrow \infty} \frac{T_{x}}{S_{T_{x}}}=\lim _{x \rightarrow \infty} \frac{T_{x}}{x+D_{x}}=\lim _{x \rightarrow \infty} \frac{T_{x}}{x}, \mathrm{P}_{\theta^{-}} \text {a.s. }
$$

The asymptotic normality (24) with condition (29) allow to use Anscombe's theorem. Thus

$$
\frac{S_{T_{x}}-T_{x} \mu_{\theta}}{\sqrt{T_{x}}} \stackrel{\mathrm{d}}{\longrightarrow} \mathcal{N}\left(0, \kappa^{\prime \prime}(\theta)\right), \text { as } x \rightarrow \infty \text {, under } \mathrm{P}_{\theta},
$$


i.e.

$$
\frac{x+D_{x}-T_{x} \mu_{\theta}}{\sqrt{T_{x}}} \stackrel{\mathrm{d}}{\longrightarrow} \mathcal{N}\left(0, \kappa^{\prime \prime}(\theta)\right), \text { as } x \rightarrow \infty, \text { under } \mathrm{P}_{\theta} .
$$

This last result with $D_{x}=\mathrm{o}(x)$, as $x \rightarrow \infty, \mathrm{P}_{\theta^{-}}$a.s., yield

$$
\frac{T_{x}-\frac{x}{\mu_{\theta}}}{\sqrt{T_{x}}} \stackrel{\mathrm{d}}{\longrightarrow} \mathcal{N}\left(0, \frac{\kappa^{\prime \prime}(\theta)}{\mu_{\theta}^{2}}\right), \text { as } x \rightarrow \infty, \text { under } \mathrm{P}_{\theta},
$$

and (25) is due to Slutski's theorem.

Lemma 3.4. Assume that the net profit condition (9) and the steepness condition (8) hold. In the short time horizon, i.e. for fixed $y<y_{r}$, we have

$$
-\frac{\log \psi(x, x y)}{x} \stackrel{x \rightarrow \infty}{\longrightarrow} l_{y}, \text { as } x \rightarrow \infty,
$$

where $y_{r}$ is given by (21) and $l_{y}$ is given by (23).

Proof of Lemma 3.4. From Lemma 3.2, $r<v_{y}$, when $y<y_{r}$, and so $\kappa\left(v_{y}\right)>0$, where $\kappa$ is given by (5), $y_{r}$ by (21), $v_{y}$ by (20) and $r$ by (14). So we have

$$
\begin{aligned}
\psi(x, x y)= & \mathrm{E}_{v_{y}}\left[\Psi\left(x, x y, v_{y}\right)\right] \\
\geq & \mathrm{E}_{v_{y}}\left[\exp \left\{-v_{y} S_{T_{x}}+T_{x} \kappa\left(v_{y}\right)\right\} ; x y-\sqrt{x} \tau\left(v_{y}\right)<T_{x} \leq x y\right] \\
= & \exp \left\{-v_{y} x+\kappa\left(v_{y}\right) x y\right\} \\
& \mathrm{E}_{v_{y}}\left[\exp \left\{-v_{y} D_{x}+\left(T_{x}-x y\right) \kappa\left(v_{y}\right)\right\} ; x y-\sqrt{x} \tau\left(v_{y}\right)<T_{x} \leq x y\right] \\
\geq & \exp \left\{-l_{y} x\right\} \mathrm{E}_{v_{y}}\left[\exp \left\{-v_{y} D_{x}-\sqrt{x} \tau\left(v_{y}\right) \kappa\left(v_{y}\right)\right\} ; x y-\sqrt{x} \tau\left(v_{y}\right)<T_{x} \leq x y\right] \\
= & \exp \left\{-l_{y} x-\kappa\left(v_{y}\right) \sqrt{x} \tau\left(v_{y}\right)\right\} \mathrm{E}_{v_{y}}\left[\exp \left\{-v_{y} D_{x}\right\} ;-1<\frac{T_{x}-\frac{x}{\mu_{v_{y}}}}{\sqrt{x} \tau\left(v_{y}\right)} \leq 0\right] \\
= & \exp \left\{-l_{y} x-\kappa\left(v_{y}\right) \sqrt{x} \tau\left(v_{y}\right)\right\}\left\{u(y)\left[\Phi(1)-\frac{1}{2}\right]+\mathrm{o}(1)\right\}, \text { as } x \rightarrow \infty,
\end{aligned}
$$

where $u(y)=\lim _{x \rightarrow \infty} \mathrm{E}_{v_{y}}\left[\exp \left\{-v_{y} D_{x}\right\}\right]$ and $\Phi$ denotes the standard normal distribution function. The asymptotic equivalence in (30) is due to Stam's Lemma, which states that $D_{x}$ and $T_{x}$ are asymptotically independent, as $x \rightarrow \infty$, and to (25) of Lemma 3.3. Thus, from (30),

$$
\liminf _{x \rightarrow \infty} \frac{\log \psi(x, x y)}{x} \geq-l_{y} .
$$

The analogous result with limsup replacing liminf and reversed inequality can be obtained in a similar way. The first details would be as follows,

$$
\begin{aligned}
\psi(x, x y) & =\exp \left\{-v_{y} x+\kappa\left(v_{y}\right) x y\right\} \mathrm{E}_{v_{y}}\left[\exp \left\{-v_{y} D_{x}+\left(T_{x}-x y\right) \kappa\left(v_{y}\right)\right\} ; T_{x} \leq x y\right] \\
& \leq \exp \left\{-l_{y} x\right\} \mathrm{E}_{v_{y}}\left[\exp \left\{-v_{y} D_{x}\right\} ; T_{x} \leq x y\right] \\
& \leq \mathrm{e}^{-l_{y} x}
\end{aligned}
$$


Remark 3.5. Lemma 3.4 can be restated as $\psi(x, x y)=\exp \left\{-x\left[l_{y}+\mathrm{o}(1)\right]\right\}$ or also as

$$
\psi(x, x y)=\xi(x, y) \mathrm{e}^{-l_{y} x}, \quad \text { as } x \rightarrow \infty,
$$

for some function $\xi: \mathbb{R}_{+} \times\left(0, y_{r}\right) \rightarrow \mathbb{R}_{+}$satisfying $\log \xi(x, y)=\mathrm{o}(x)$.

The next result is the direct generalization of Esscher's approximation for the compound Poisson sum, see e.g. p. 170 in Asmussen and Glynn (2007), to the considered Lévy processes.

Lemma 3.6. Assume that the steepness condition (8) holds. Then for fixed $y>0$, we have

$$
\zeta(x, x y) \sim \frac{1}{v_{y} \sqrt{2 \pi x y \kappa^{\prime \prime}\left(v_{y}\right)}} \mathrm{e}^{-l_{y} x}, \quad \text { as } x \rightarrow \infty,
$$

where $l_{y}$ is given by (23) and $v_{y}$ by (20).

Proof of Lemma 3.6. From (12) with $A=\left\{S_{t} \geq x\right\}$, we obtain

$$
\begin{aligned}
\zeta(x, t) & =\mathrm{E}_{\theta}[\mathrm{Z}(x, t, \theta)] \\
& =\mathrm{E}_{\theta}\left[\exp \left\{-\theta S_{t}+t \kappa(\theta)\right\} ; S_{t} \geq x\right] \\
& =\exp \{-\theta x+t \kappa(\theta)\} \mathrm{E}_{\theta}\left[\exp \left\{-\theta \sqrt{t \kappa^{\prime \prime}(\theta)} \frac{S_{t}-x}{\sqrt{t \kappa^{\prime \prime}(\theta)}}\right\} ; \frac{S_{t}-x}{\sqrt{t \kappa^{\prime \prime}(\theta)}} \geq 0\right] .
\end{aligned}
$$

Consider $t=x y$ and (24) with $\theta=v_{y}$. We find $t \mu_{v_{y}}=x$ and thus (32) yields, as $x \rightarrow \infty$,

$$
\begin{aligned}
\zeta(x, x y) & \sim \exp \left\{-v_{y} x+x y \kappa\left(v_{y}\right)\right\} \frac{1}{\sqrt{2 \pi}} \int_{0}^{\infty} \exp \left\{-v_{y} \sqrt{x y \kappa^{\prime \prime}\left(v_{y}\right)} z\right\} \mathrm{e}^{-\frac{z^{2}}{2}} \mathrm{~d} z \\
& =\frac{1}{v_{y} \sqrt{2 \pi x y \kappa^{\prime \prime}\left(v_{y}\right)}} \exp \left\{-x\left[v_{y}+y \kappa\left(v_{y}\right)\right]\right\} \int_{0}^{\infty} \mathrm{e}^{-z} \underbrace{\exp \left\{-\frac{1}{2} \frac{z^{2}}{v_{y}^{2} x y \kappa^{\prime \prime}\left(v_{y}\right)}\right\}}_{\stackrel{x \rightarrow \infty}{\longrightarrow} 1} \mathrm{~d} z .
\end{aligned}
$$

Monotone convergence yields (31).

Remark 3.7. From Remark 3.5 and Lemma 3.6, it can be confirmed that the probability $\check{\psi}(x, t)$ refers indeed to a rare event. Indeed, for $t=x y$,

$$
\begin{aligned}
\check{\psi}(x, x y) & =\psi(x, x y)-\zeta(x, x y) \\
& =\left(\xi(x, y)-\frac{1}{v_{y} \sqrt{2 \pi x y \kappa^{\prime \prime}\left(v_{y}\right)}}\right) \mathrm{e}^{-l_{y} x} \\
& \stackrel{x \rightarrow \infty}{\longrightarrow} 0,
\end{aligned}
$$

because $\xi(x, y)=\mathrm{e}^{\mathrm{o}(x)}$. 
We can now present a proof of logarithmic efficiency of the importance sampling estimator $\check{\Psi}\left(x, t, v_{y}\right)$ of the probability of ruin with recuperation.

Proof of Theorem 3.1. For $t=x y$, we have

$$
\begin{aligned}
\mathrm{E}_{v_{y}}\left[\left\{\Psi\left(x, t, v_{y}\right)-\mathrm{Z}\left(x, t, v_{y}\right)\right\}^{2}\right]= & \mathrm{E}_{v_{y}}\left[\Psi^{2}\left(x, t, v_{y}\right)\right]+\mathrm{E}_{v_{y}}\left[\mathrm{Z}^{2}\left(x, t, v_{y}\right)\right] \\
& -2 \underbrace{\mathrm{E}_{v_{y}}\left[\Psi\left(x, t, v_{y}\right) \mathrm{Z}\left(x, t, v_{y}\right)\right]}_{\geq 0} \\
\leq & \mathrm{E}_{v_{y}}\left[\Psi^{2}\left(x, t, v_{y}\right)\right]+\mathrm{E}_{v_{y}}\left[\mathrm{Z}^{2}\left(x, t, v_{y}\right)\right],
\end{aligned}
$$

where $\Psi(x, t, \theta)$ is given by $(15)$ and $\mathrm{Z}(x, t, \theta)$ by (16). We also have

$$
\begin{aligned}
\mathrm{E}_{v_{y}}\left[\Psi^{2}\left(x, t, v_{y}\right)\right] & =\mathrm{e}^{-2 l_{y} x} \mathrm{E}_{v_{y}}\left[\exp \left\{2[\underbrace{-v_{y} D_{x}}_{\leq 0}+\underbrace{\left(T_{x}-x y\right) \kappa\left(v_{y}\right)}_{\leq 0}]\right\} ; T_{x} \leq x y\right] \\
& \leq \mathrm{e}^{-2 l_{y} x} .
\end{aligned}
$$

Following similar steps as in the proof of Lemma 3.5, we can show

$$
\begin{aligned}
\mathrm{E}_{v_{y}}\left[\mathrm{Z}^{2}\left(x, t, v_{y}\right)\right] & \sim \frac{1}{2 v_{y} \sqrt{2 \pi x y \kappa^{\prime \prime}\left(v_{y}\right)}} \exp \left\{-2 x\left[v_{y}+y \kappa\left(v_{y}\right)\right]\right\} \\
& =\frac{1}{4 v_{y} \sqrt{\pi x y \kappa^{\prime \prime}\left(v_{y}\right)}} \mathrm{e}^{-2 l_{y} x}, \quad \text { as } x \rightarrow \infty .
\end{aligned}
$$

As mentioned in Remark 3.7, we have

$$
\psi(x, t)-\zeta(x, t) \sim\left(\xi(x, y)-\frac{1}{\sqrt{2 \pi x y \kappa^{\prime \prime}\left(v_{y}\right)} v_{y}}\right) \mathrm{e}^{-l_{y} x}, \quad \text { as } x \rightarrow \infty .
$$

By considering all results above and $x$ sufficiently large, we obtain

$$
\begin{aligned}
& \frac{\mathrm{E}_{v_{y}}\left[\left\{\Psi\left(x, x y, v_{y}\right)-\mathrm{Z}\left(x, x y, v_{y}\right)\right\}^{2}\right]}{\{\psi(x, x y)-\zeta(x, x y)\}^{2-\epsilon}} \\
& \quad \leq\left(1+\frac{1}{4 v_{y} \sqrt{\pi x y \kappa^{\prime \prime}\left(v_{y}\right)}}\right)\left(\xi(x, y)-\frac{1}{v_{y} \sqrt{2 \pi x y \kappa^{\prime \prime}\left(v_{y}\right)}}\right)^{\varepsilon-2} \mathrm{e}^{-\varepsilon l_{y} x} \\
& \quad=\exp \left\{-\left[\varepsilon l_{y}+(\varepsilon-2) \mathrm{o}(1)\right] x\right\} \\
& \quad \stackrel{x \rightarrow \infty}{\longrightarrow} 0, \quad \forall \varepsilon>0,
\end{aligned}
$$

because $\xi(x, y)=\mathrm{e}^{\mathrm{o}(x)}$. This is the desired logarithmic efficiency, according to (19).

\section{Final remarks}

We conclude this article with two final remarks relating the proposed logarithmic efficient estimator of Theorem 3.1 with saddlepoint approximations and with an importance sampling algorithm proposed in the context of pricing double barrier financial options. 
For the particular situation where the loss process $S$ is a compound Poisson process perturbed by a Wiener process, the desired quantity $\check{\psi}(x, t)$ can be alternatively computed by the saddlepoint approximation to $\psi(x, t)$ suggested by Gatto and Baumgartner (2014), together with the saddlepoint approximation to $\zeta(x, t)$ of Gatto (2010). Saddlepoint approximations are substantially faster to compute than importance sampling, although they are conceptually more sophisticated and by far less popular than Monte Carlo methods.

The following importance sampling scheme, for pricing the so-called down-and-in barrier option or digital knock-in option, is due to Boyle et al. (1997), see also Glassermann (2003), p. 264-267. Assume $S=\left\{S_{t}\right\}_{t \geq 0}$ in $\mathbb{R}_{+}^{[0, \infty)}$ represents underlying asset price, where $x=S_{0}>0$ is fixed. This barrier option with time horizon $[0, t]$, for some $t>0$, has payoff

$$
\Xi(t)=\mathrm{I}\left\{S_{t}>k_{2}, \min _{1 \leq k \leq m-1} S_{t_{k}}<k_{1}\right\},
$$

where $0 \leq k_{1} \leq x \leq k_{2}<\infty$ are fixed values ( $k_{2}$ is called strike) and $t_{0} \stackrel{\text { def }}{=} 0<t_{1}<$ $\ldots<t_{m-1}<t_{m} \stackrel{\text { def }}{=} t$. The quantity of interest is the payoff probability $\xi(t)=\mathrm{E}[\Xi(t)]$, which is often small because $k_{1}$ is typically substantially smaller than $x$. Thus this situation generalizes the insurer's ruin with recuperation, where $k_{1}=0$. Given $X_{1}, \ldots, X_{m}$, independent and identically distributed, $X_{0} \stackrel{\text { def }}{=} 0$ and $Y_{k}=\sum_{j=0}^{k} X_{j}$, for $k=0, \ldots, m$, it is assumed $S_{t_{k}}=x \exp \left\{Y_{k}\right\}$, for $k=0, \ldots, m$. Denote by $T$ is the first index or time when $\left\{Y_{k}\right\}_{k=0, \ldots, m}$ falls under $-b \stackrel{\text { def }}{=} \log \left(k_{1} / x\right)$. An importance sampling estimator is based on a double exponential tilt under which the process $\left\{Y_{k}\right\}_{k=0, \ldots, m}$ receives: a negative drift from time 0 and until when it reaches level $-b$, i.e. until stopping time $T$, and a positive drift from $T$ until $m$. Thus, likelihood ratio involves two exponential tilt parameters: the first one imposing negative drift over $\{0, \ldots, T-1\}$ and the second one imposing positive drift over $\{T, \ldots, m\}$. The choice of these two tilting parameters is not (directly) based on fulfillments of criteria (17) or (18). It is rather dictated by these redrifting constraints with another condition aiming to eliminate the main source of variability from the likelihood ratio, which is $T$. The resulting equations defining the two tilting parameters admit simple closed form solutions when $S$ is a geometric Browian motion.

\section{$5 \quad$ References}

Applebaum, D. (2004), Lévy Processes and Stochastic Calculus, Cambridge.

Asmussen, S. (1985), "Conjugate processes and the simulation of ruin problems", Stochastic Processes and their Applications, 20, 213-229.

Asmussen, S. (2000), Ruin Probabilities, World Scientific. 
Asmussen, S., Glynn, P. W. (2007), Stochastic Simulation. Algorithms and Analysis, Springer.

Avram, F., Palmowski, Z., Pistorius, M. R. (2007), "On the optimal dividend problem for a spectrally negative Lévy process", Annals of Applied Probability, 17, 156-180.

Bertoin, J. (1996), Lévy Processes, Cambridge University Press.

Biffis, E., Morales, M. (2010), "On a generalization of the Gerber-Shiu function to path dependent penalties", Insurance: Mathematics and Economics, 46, 92-97.

Boyle, P., Broadie, M., Glasserman, P. (1997), "Monte Carlo methods for security pricing", Journal of Economic Dynamics and Control, 21, 1267-1321.

Bucklew, J. A. (2004), Introduction to Rare Event Simulation, Springer.

Gatto, R. (2010), "A saddlepoint approximation to the distribution of inhomogeneous discounted compound Poisson processes", Methodology and Computing in Applied Probability, $12,533-551$.

Gatto, R. (2014), "Importance sampling approximations to various probabilities of ruin of spectrally negative Lévy risk processes", Applied Mathematics and Computation, 243, 91-104.

Gatto, R., Baumgartner, B. (2014), "Saddlepoint approximations to the probability of ruin in finite time for the compound Poisson risk process perturbed by diffusion", Methodology and Computing in Applied Probability, 16, 561-582.

Glasserman, P. (2003), Monte Carlo Methods in Financial Engineering, Springer.

Klüppelberg, C., Kyprianou, A. E., Maller, R. A. (2004), "Ruin probabilities and overshoots for general Lévy insurance risk processes", Annals of Applied Probabability, 14, 1766-1801. Kyprianou, A. E., Palmowski, Z. (2007), Distributional study of de Finettis dividend problem for a general Lévy insurance risk process, Journal of Applied Probability, 44, 428-443.

Siegmund, D. (1976), "Importance sampling in the Monte Carlo study of sequential tests", The Annals of Statistics, 4, 673-684. 